\title{
Microfluidic Tools for High-Throughput Screening
}

\author{
Todd A. Thorsen
}

Department of Mechanical Engineering, Massachusetts Institute of Technology, Cambridge, MA, USA

High-throughput screening (HTS) is an established technology in the pharmaceutical industry (1). Over the past decade, there has been a logarithmic increase in the industry's ability to screen large combinatorial libraries of compounds against target molecules (2). The technology to achieve this has come in the form of robotics, high-density microplates, small volume (microliter) liquid handling, and sophisticated detection schemes. Significant effort has gone into the design of HTS workstations capable of screening tens of thousands of compounds in a 24-h period. However, these workstations come with a large price tag, ranging from several hundred thousand to millions of dollars.

A considerable amount of work is necessary to optimize nascent microfluidic devices for HTS applications. Stateof-the-art commercial microfluidic devices are principally made by the micromachining of silicon (3) and glass and rely on electroosmotic flow $(4,5)$ to drive liquid through the channels, requiring high salt concentrations and a voltage source. This process generates gas bubbles, creating ionic conditions that are far from ideal for assays measuring enzymatic activity or protein-protein interactions. Other problems with hard polymer microfluidic devices include the need to build up layers to efficiently seal the channel networks, making layer-layer adhesion a serious concern during the fabrication process, and the lack of a good compartmentalization technology for the large-scale analysis of chemical or biological libraries.

In thinking about a generic design for microfluidic HTS devices for catalytic screening applications that confine both enzyme and substrate to picoliter volumes, both serial and parallel approaches can be explored. Using a serial strategy, each compound of interest is screened sequentially using a common microfluidic channel with a single detection element. Mechanically, throughput depends on factors such as flow speed, sample concentration, and the acquisition time of the detector. In contrast, parallel screening functions like an ultra-high density microplate, in which thousands of compounds are arrayed into individual picoliter-scale compartments, with a detector element that probes the entire matrix. Throughput is principally limited by the number of compartments in the array. We have designed and developed microfluidic chips employing both serial and parallel screening strategies. Unlike in state-ofthe-art microfluidic devices relying on electroosmotic flow, fluidic trafficking and compartmentalization in our chips are pressure-based, using integrated elastomeric valves whose function is independent of solvent composition.

\section{Droplet-Generating Microfluidic Devices for Soluble Assays}

Encapsulation of biological agents is not a new concept for the pharmaceutical industry. For the past two decades, liposomes have been used as carriers for a wide variety of drugs, including anti-tumor and anti-fungal compounds, as well as genetic drugs, such as antisense oligonucleotides and plasmids for gene therapy (6). However, liposomes are difficult to prepare, requiring organic solvents to hydrate the dried lipid mixture and techniques, such as sonication or French press, to size the final liposomes. Problems with the final product include size heterogeneity and poor aqueous encapsulation rates, often as low as $20 \%$ (7). Emulsions, consisting of water, oil, and a surfactant, are an alternative to liposomes. Depending on the relative concentration of each component, emulsions can exist as micelles consisting of surfactant-stabilized oil droplets in an aqueous solution or as reverse micelles in which water droplets are present in a bulk oil solution. While reverse micelles have the advantage over liposomes of encapsulating $100 \%$ of the aqueous phase, synthesis of homogeneous emulsions has been considered a black art (8). Recognizing their potential as miniature bioreactors, we have developed microfluidic devices capable of producing highly homogeneous emulsions.

In recent work, we introduced a new emulsification technology utilizing elastomer-based microfluidic devices (9). The microfluidic devices, consisting of optically transparent polydimethylsiloxane (PDMS), are cast from silicon wafer molds containing positive-relief channels by using standard lithographic techniques. Within these devices, pressurized immiscible fluids are mixed at an orthogonal junction of two microchannels, combining cross-flow and viscous shear to generate monodisperse water-in-oil (W/O) droplets with picoliter-scale volumes [coefficient of variation (cv) $<5 \%$ ] (Figure 1). The addition of nonionic surfactants adds stability to the formed droplets. By modifying the relative pressures of the two fluids, the size of the formed droplets can range from nanometers to microns in diameter. Capable of generating thousands of droplets per second, these devices are being optimized for high-throughput enzymatic screening assays, dynamically encapsulating single cells expressing recombinant enzyme and substrate in each droplet.

Mixing in the droplets confined in the microchannels, with typical diameters of $10-20 \mu \mathrm{m}$, is highly efficient. Flow is laminar in the microfluidic chips, preventing mixing of the aqueous components until after encapsulation. However, after formation, the aqueous droplets suspended in the bulk oil solution contact the top and bottom microchannel walls as they flow toward the outlet. In a straight microfluidic channel, this contact sets up in the droplet a bilateral circulating flow field, which resembles two counter-rotating vortices. By introducing bends into the devices, the streamlines in these vortices fold over on each other, resulting in rapid mixing of the droplet contents. Depending on the droplet flow rate and the number of bends in the devices, mixing times on the millisecond time scale have been reported for dyes like Fluo-4 (10). With rapid mixing dynamics, these devices have potential applications not only in end point assays but also for real-time monitoring of enzyme kinetics at the single-cell level. 


\section{Techniques Essay}

\section{Addressable Microfluidic Array Devices}

Primarily for HTS applications, clever array-based micropatterning and compartmentalization systems for cells and proteins have been established by a variety of techniques. Examples include polydimethylsiloxane (PDMS) membranes with molded pores for the deposition of cell and protein arrays onto glass substrates $(11,12)$, the patterning of biomolecules using elastomeric stamps $(13,14)$, and nanoliter-scale assay plates (15).

We have used multilayer soft lithography (16) to make array-based microfluidic devices with thousands of individually addressable chambers (Figure 2). Microchannels on the bottom of the devices, when sealed to a glass coverslip.

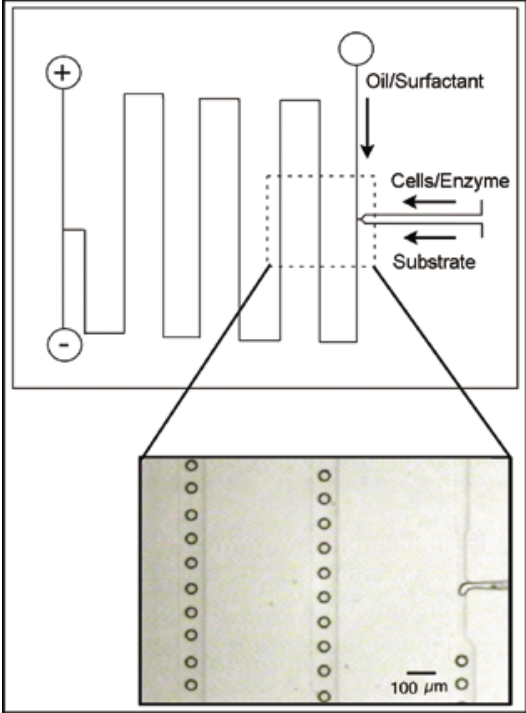

Figure 1. Droplet-generating biochemical screening chip. Monodispersed water droplets maintain uniform spacing and periodicity as they flow towards the device outlet. serve as conduits

for biological material whose flow is regulated by integrated elastomeric valves. The devices are initially fabricated as two distinct silicone layers on microchannel-patterned silicon wafers. The thick silicone layer containing the channels required to actuate the valves (control channels) is covalently bonded to a thin layer of silicone containing the channels that will be filled with biological reagents (flow channels), such that a silicone membrane with a thickness of approximately $30 \mu \mathrm{m}$ separates the control and flow channels at the points where they overlap. The membrane in the crossover regions functions as a valve, in which pneumatic pressure applied to the control channels causes the elastomeric membrane to deflect down, closing off the underlying flow channels (Figure 3 ). Pneumatic actuation of the valves is accomplished through interconnect holes punched through the thick layer prior to the secondary bonding step, connecting the isolated control channels to a pressurized air supply controlled by miniature solenoid valves, while interconnect holes on the bottom flow layer provide access for sample input and output. Specific actuation of these valve arrays using computer software creates an elaborate fluidic trafficking system, in which individual cells can be independently compartmentalized, assayed, and recovered from the devices (17).

\section{Future Applications and Challenges}

Increasing biocomplexity created by explosive advances in genomics and proteomics in the last decade has established the need for HTS technologies to transform the vast collections of data generated from these systems into organized knowledge (18). Elastomeric microfluidic chips are poised to fill this role, using ultra-low sample volumes and multiplexing technology to provide high-density, addressable screening platforms for both in vitro and cell-based applications. As we perfect the manufacture procedure for

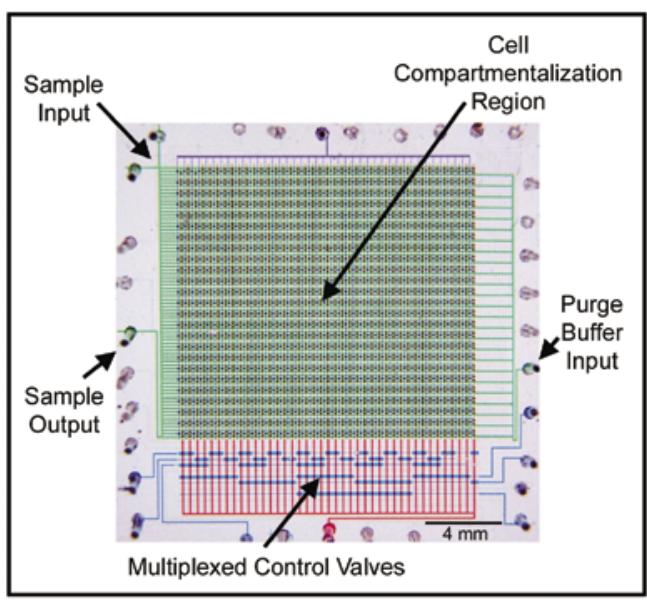

Figure 2. Silicone microfluidic array chip for high-throughput single cell assays. Chip contains 1000 independent compartments and 3574 elastomeric valves.

these devices, the future challenges lie in adapting biological protocols for nanoliter-scale assays.

For applications like single-cell enzymatic assays, expression variability, which is dependent on factors like the stage of a cell in its growth cycle and the difficulty of expressing the protein of interest, must be taken into consideration. This additional noise, which is averaged out in microplate-based assays that measure the average activity of group of cells, makes selection of mutants with small improvements in activity more difficult to identify. However, high-throughput-based microfluidic devices, which can rapidly screen thousands of cells in either a serial or parallel format, may be able to identify "super" mutants
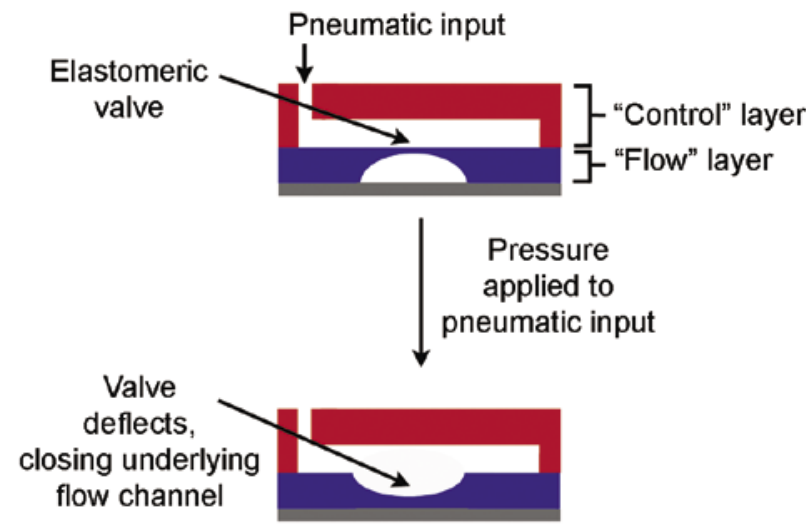

Figure 3. Cross-section of silicone microfluidic chip illustrating valve mechanics. In the regions where the flow and control channels crossover, the thin silicone membrane that separates them is deflected under pneumatic pressure, closing off the underlying flow channel. 


\section{Techniques Essay}

in a recombinant protein library $\left(10^{5}-10^{7}\right)$ that may never be found by techniques that only sample a small fraction of the library diversity. Without the need to isolate, grow, and induce cells in individual wells of microplates, integrated microfluidic devices can reduce the screening process from days to hours, depending on the assay parameters. While much of the research is still at the proof-of-concept stage, microfluidics has the potential to transform biochemical analysis in the same way that miniaturization transformed computing, making tools that are smaller, more integrated, less expensive, and a lot faster (19).

\section{Acknowledgments}

The author would like to thank Dr. Stephen Quake at Caltech for helpful discussions.

\section{References}

1.Battersby, B.J. and M. Trau. 2002. Novel miniaturized systems in high-throughput screening. Trends Biotechnol. 20:167-173.

2.Beggs, M. 2001. HTS—where next? Drug Discovery World 2:125-134.

3.Martynova, L., L.E. Locasico, M. Gaitan, G.W. Kramer, and R.G. Christiansen. 1997. Fabrication of plastic microfluid channels by imprinting methods. Anal. Chem. 69:4783-4789.

4.Chou, H.-P., C. Spence, A. Scherer, and S. Quake. 1999. A microfabricated device for sizing and sorting DNA molecules. Proc. Natl. Acad. Sci. USA 96:11-13.

5.Chou, H.-P., C. Spence, A. Scherer, and S. Quake. 1998. A microfabricated device for sizing DNA and sorting cells. Proc. SPIE 3258:181.

6.Maurer, N., D.B. Fenske, and P.R. Cullis. 2001. Developments in liposome drug delivery systems. Expert Opin. Biol. Ther. 1:923-947.

7.Hauser, H. 1987. Spontaneous vesiculation of uncharged phospholipid dispersions consisting of lecithin and lysolecithin. Chem. Phys. Lipids 43:283-299.
8.Tawfik, D.S. and A.D. Griffiths. 1998. Man-made cell-like compartments for molecular evolution. Nat. Biotechnol. 16:652-656.

9.Thorsen, T., R.W. Roberts, F.H. Arnold, and S.R. Quake. 2001. Dynamic pattern formation in a vesicle-generating microfluidic device. Phys. Rev. Lett. 86:4163-4166.

10.Song, H., J.D. Tice, and R.F. 2003. Ismagilov. A microfluidic systems for controlling reaction networks in time. Angew. Chem. Intl. Ed. 42: 768-772

11.Ostuni, E., R. Kane, C.S. Chen, D.E. Ingber, and G.M. Whitesides. 2000. Patterning mammalian cells using elastomeric membranes. Langmuir 16:7811-7819.

12.Griscom, L., P. Degenaar, B. LePioufle, E. Tamiya, and H. Fujita. 2001. Cell placement and neural guidance using a three-dimensional microfluidic array. Jpn. J. Appl. Physics 40:5485-5490.

13. James, C.D., R.C. Davis, L. Kam, H.G. Craighead, M. Isaacson, J.N. Turner, and W. Shain. 1998. Patterned protein layers on solid substrates by thin stamp microcontact printing. Langmuir 14:741-744.

14.Lahiri, J., E. Ostuni, and G.M. Whitesides. 1999. Patterning ligands on reactive SAMs by microcontact printing. Langmuir 15:2055-2060.

15.Mere, L., T. Bennett, P. Coassin, P. England, B. Hamman, T. Rink, S. Zimmerman, and P. Negulescu. 1999. Miniaturized FRET assays and microfluidics: key components for ultra-high-throughput screening. Drug Discov. Today 4:363-369.

16.Unger, M.A., H.-P. Chou, T. Thorsen, A. Scherer, and S. Quake. 2000. Monolithic microfabricated valves and pumps by multilayer soft lithography. Science 288:113-116.

17.Thorsen, T., S.J. Maerkl, and S.R. Quake. 2002. Microfluidic largescale integration. Science 298:580-584.

18. Khandurina, J. and Andras Guttman. 2002. Microchip-based highthroughput screening analysis of combinatorial libraries. Curr. Opin. Chem. Biol. 6:359-366

19.Sassi, A.P., Q.F. Xue, and H.H. Hooper. 2000. Making analysis in the life sciences faster through miniaturization. Am. Lab. 32:36-41. 